\title{
Avaliação dos riscos envolvidos na produção de milho e soja nos municípios do Mato Grosso
}

Evaluation of the risks involved in the corn and soybeans productions in Mato Grosso's cities

\author{
Fernanda Aparecida Castro Pereira ${ }^{1 *}$; Aline Fernanda Soares ${ }^{2}$
}

\author{
1 Escola Superior de Agricultura Luiz de Queiroz da Universidade de São Paulo - Doutora em Genética e \\ Melhoramento de Plantas e Especialista em Agronegócio - Av. Pádua Dias, 11 - CEP: 13418-900 - \\ Piracicaba (SP), Brasil \\ 2 Escola Superior de Agricultura Luiz de Queiroz da Universidade de São Paulo - Departamento de \\ Economia, Administração e Sociologia, Av. Pádua Dias, 11, CEP 13418-900, Piracicaba (SP), Brasil
}

\section{Resumo}

A agricultura opera com instabilidade de produção principalmente devido a fatores imprevisíveis que afetam a cultura. Por esse motivo, as seguradoras têm dificuldade de quantificar o risco exato associado aos municípios produtores, principalmente no estado de Mato Grosso. Não existem estudos claros associados à segmentação e quantificação desse risco em uma escala menor. Diante desse fato, o trabalho buscou quantificar e segmentar o risco de produção de milho e soja no estado Mato-Grossense por meio de análises de agrupamento. Para o agrupamento das médias, foi adotada a metodologia não hierárquica de agrupamento denominada K-means, onde se obteve sete clusters. O risco associado à cada cluster foi estimado com base no cálculo do coeficiente de variação da média de produtividade de milho e soja entre os municípios. O trabalho apresentou variação na taxa de risco entre os clusters principalmente considerando a produtividade de milho; os municípios do cluster número 3 (Porto Alegre do Norte, Serra Nova Dourada, Santa Terezinha, Jangada, Barra do Bugres e Porto Estrela) apresentaram as maiores taxas de risco (10,2\%) e podem ser desconsiderados da carteira de crédito das seguradoras; e, para a soja, as taxas de riscos entre os clusters foram similares devido à estabilidade produtiva até o momento. Nesse cenário, sugere-se que as seguradoras trabalhem com prêmios menores e introduzam programas de subvenção ao seguro rural naqueles municípios com risco individual elevado.

Palavras chaves: análise de agrupamento, produtividade agrícola, seguro agrícola

\section{Abstract}

Agriculture operates with instability of production mainly due to unpredictable factors affecting the culture. Therefore, insurances have been difficult to quantify the exact risk associated with agricultural producing in the cities, mainly in the state of Mato Grosso. There are no clear studies associated with segmentation and quantification of this risk on a smaller scale. Therefore, the objective of this research was to quantify the risk and target production of corn and soybeans in Mato Grosso by methods of cluster analysis. For the means grouping, it was adopted the methodology of non-hierarchical clustering called K-means and seven clusters were obtained. The risk associated within each cluster was based on the coefficient of variation of the corn and soybeans yield among the cities. There was variation in the risk among clusters especially considering the corn yield. The cities Porto Alegre do Norte, Serra Nova Dourada, Santa Terezinha, Jangada, Barra do Bugres e Porto Estrela from the cluster number 3 had the highest risk rates and may be disregarded from the insurance credit portfolio. For soybeans, the risk rates among clusters were similar due to production stability so far. This scenario presented by the research suggests that insurers work with lower premiums values and introduce subsidy programs for rural insurance in those cities with high individual risk.

Keywords: agricultural insurance, agricultural yield, cluster analysis

\section{Introdução}

\footnotetext{
"Autor correspondente:<fernandacpereira01@gmail.com> Enviado: 12 dez. 2017 Aprovado: 26 abr. 2017
} 
O estado de Mato Grosso é o maior estado em extensão territorial da região e o terceiro maior do país. Atualmente, o crescimento na região de Sinop, Sorriso, Nova Mutum, Lucas do Rio Verde e Matupá se mostra um forte propulsor para o desenvolvimento econômico do estado, baseado na produção e venda de grãos. Em 2016, o Mato Grosso liderou como maior produtor nacional de grãos, com uma participação de $23,9 \%$. Sendo o principal produtor de milho em grão $2^{\underline{a}}$ safra, participando com $38,6 \%$ desta produção nacional. Para a soja o estado obteve, em 2016, uma produção de 26,3 milhões de toneladas, e a previsão é de um aumento de 10,6\% em relação a 2016 (IBGE, 2016).

As atividades agrícolas estão sujeitas a uma vasta gama de riscos e incertezas, devido aos fatores econômicos e ao ambiente em que a agricultura opera. Dentre os fatores econômicos, pode-se citar a possibilidade de descasamento do fluxo de caixa, uma vez que a compra dos insumos e a produção ocorrem num período anterior à venda do produto final - a produção de madeira de eucalipto, por exemplo, tem um ciclo superior a um ano. Quanto ao ambiente em que a agricultura opera, fatores climáticos adversos, pragas e doenças podem afetar negativamente a produção, causando graves prejuízos econômicos aos produtores (Ullah et al., 2015). É de conhecimento comum que o clima representa a principal fonte de incerteza na produção agrícola, rara as exceções.

Estima-se que 93\% das perdas na safra de soja do Rio Grande do Sul ocorram em razão das estiagens (Berlato e Fontana, 1999). Na última safra, a perda por estiagem chegou a $20 \%$ também no Mato Grosso. A projeção da Companhia Nacional de Abastecimento [CONAB] foi de 57,13 milhões de toneladas para a safrinha de milho de 2016 entretanto o esperado pelos agricultores é algo abaixo de 55 milhões. Nos principais estados produtores, as perdas já superaram os 10\% (CONAB, 2016). Tradicionalmente, os agricultores tentam se proteger contra variações de produtividade relacionadas com o clima através da compra de seguros agrícolas (Musshoff et al., 2011).

$\mathrm{Na}$ cultura da soja e do milho, o produtor tem utilizado o zoneamento agroclimático como uma ferramenta na gestão de riscos e constitui-se numa ferramenta de fundamental importância em várias atividades do setor agrícola. Fruto de um trabalho multi-institucional e multidisciplinar, o zoneamento agroclimático da cultura da soja procurou delimitar as áreas com maior aptidão climática para 0 desenvolvimento da cultura, visando à obtenção de maiores rendimentos e menores riscos (Favarin e Oliveira, 2013). 
Além do zoneamento, os produtores têm utilizado instrumentos oferecidos pelo setor de seguros e pelo mercado financeiro (mercados futuros, de opções, contratos a termo, etc.), além da troca de insumos por produtos. O seguro agrícola é um dos mecanismos mais eficazes para transferir o risco para outros agentes econômicos. Por meio dele, um indivíduo transfere uma despesa futura e incerta (dano), de valor elevado, por uma despesa antecipada e certa de valor relativamente menor (prêmio) (Ozaki, 2008). Em dezembro de 2003, o Congresso Nacional do Brasil aprovou a Lei no 10.823 que autorizou a concessão de subvenção econômica ao prêmio do seguro rural, regulamentada pelo Decreto no 5.121/04. O Programa de Subvenção ao Prêmio do Seguro Rural [PSR] oferece ao agricultor a oportunidade de segurar sua produção com custo reduzido, por meio de auxílio financeiro do governo federal e estadual, como é o caso do estado de São Paulo, permitindo a complementação de valores por subvenções (MAPA, 2016).

A ocorrência incerta de eventos catastróficos ligados ao clima dificulta 0 trabalho das companhias seguradoras no ramo de atividade. Isso porque há uma imprevisibilidade dos fatos e, como consequência, observa-se que o risco é altamente correlacionado entre unidades seguradas quando determinado fenômeno climático atinge várias propriedades. Esse fato viola um dos princípios básicos do mercado de seguros: as unidades expostas devem ser homogêneas e independentes (Ozaki, 2007). Esta condição de segurabilidade diz que as unidades seguradas devem estar geograficamente dispersas. Ou seja, quando os sinistros são independentes e identicamente distribuídos, o risco agregado se torna menor do que o risco individual.

Para o seguro agrícola, a quantificação municipalizada do risco é fundamental para que as seguradoras possam trabalhar com risco diversificado e precificar adequadamente os contratos. Ozaki, (2009) quantificou esse risco por meio de uma análise de agrupamento. Como técnica multivariada tem por objetivo reunir as observações em grupos, de tal forma que os elementos de um mesmo grupo sejam semelhantes (Pohlmann, 2007).

Ao segmentar a mesorregião trabalha-se com subpopulações mais homogêneas, devido à redução da variância entre os agrupamentos, fato que possibilita uma gestão mais eficiente do risco.

Os métodos de segmentação são denominados também de métodos de agrupamento. Esses métodos consistem em técnicas computacionais a fim de separar objetos em grupos, baseando-se nas características que estes objetos possuem. A ideia básica consiste em colocar em um mesmo grupo objetos que sejam similares de 
acordo com algum critério pré-determinado (Frei, 2006). Existe, por exemplo, o método K-means. Esse método parte de um número de grupos (clusters) definido a priori (k) e calcula pontos que representam os centros destes grupos e que são espalhados de forma homogênea no conjunto de respostas obtidas e movidos, heuristicamente, até alcançar um equilíbrio estático. Procede-se a uma divisão de todos os casos obtidos pelos $\mathrm{k}$ grupos preestabelecidos e a melhor partição dos $\mathrm{n}$ casos será aquela que otimiza o critério escolhido (Everitt et al., 2011).

Devido à importância de Mato Grosso no cenário nacional da produção de soja e milho, este trabalho teve como objetivo estudar o comportamento produtivo dos municípios do Mato Grosso produtores de milho e soja, agrupar aqueles municípios com características semelhantes de produtividade e quantificar o risco associado à cada grupo formado.

\section{Material e Métodos}

A região de estudo corresponde à área produtiva de milho e soja do estado de Mato Grosso. Por meio do Instituto Brasileiro de Geografia Estatística [IBGE], foram coletados dados de produtividade de milho e soja em $\mathrm{kg} \mathrm{ha}^{-1}$ de 92 municípios produtores entre os anos de 2000 e 2013 (IBGE, 2015). Todas as análises foram realizadas no programa $\mathrm{R}$ com o auxílio do pacote Stats versão 3.4 .0 e os gráficos no programa Quantum GIS [QGIS] versão 2.2.0.

Para o agrupamento das médias, foi adotada a metodologia não hierárquica de agrupamento denominada K-means, onde o número de clusters (7) foi pré-definido com o auxílio de um dendograma, obtido pelo método de Ward, que minimiza o quadrado da distância euclidiana às médias dos grupos. A distância euclidiana (Manly, 1986) está presente na eq. (1).

$$
d=\sum_{j=l}^{p}\left\{\frac{\left(X_{i j}-X_{i^{*} j^{*}}\right)^{2}}{X_{i j}}\right\}
$$

onde, $p$ : número de variáveis; $X_{i j}$ : média indivíduo do município $i$ na variável $j$

O dendograma é um método hierárquico de agrupamento, que permite ter uma visão bidimensional da similaridade ou dissimilaridade de todo o conjunto de amostras utilizado no estudo. Nesse caso, foi utilizado o método de Ward (Hair et al., 2005) para a confecção do dendograma, que consiste em um procedimento de agrupamento hierárquico no qual a medida de similaridade usada para juntar agrupamentos é calculada como a soma de quadrados entre os dois agrupamentos feita sobre todas as 
variáveis. Esse método tende a resultar em agrupamentos de tamanhos aproximadamente iguais devido a minimização da variância dentro dos grupos (clusters) (Hair et al., 2005).

A taxa de risco associado aos clusters foi obtido por meio do cálculo do coeficiente de variação [CV] das médias de produtividade de cada cluster tanto para soja quanto para o milho, eq. (2).

$$
C V(\%)=\frac{s}{\bar{x}}
$$

onde, s: desvio padrão da média; $\bar{x}$ : média das observações

\section{Resultados e Discussão}

O estado brasileiro de Mato Grosso é dividido em cinco mesorregiões geográficas: Centro-Sul Mato-Grossense; Nordeste Mato-Grossense; Norte MatoGrossense; Sudeste Mato-Grossense e Sudoeste Mato-Grossense. Essas mesorregiões estão identificadas na Figura 1.

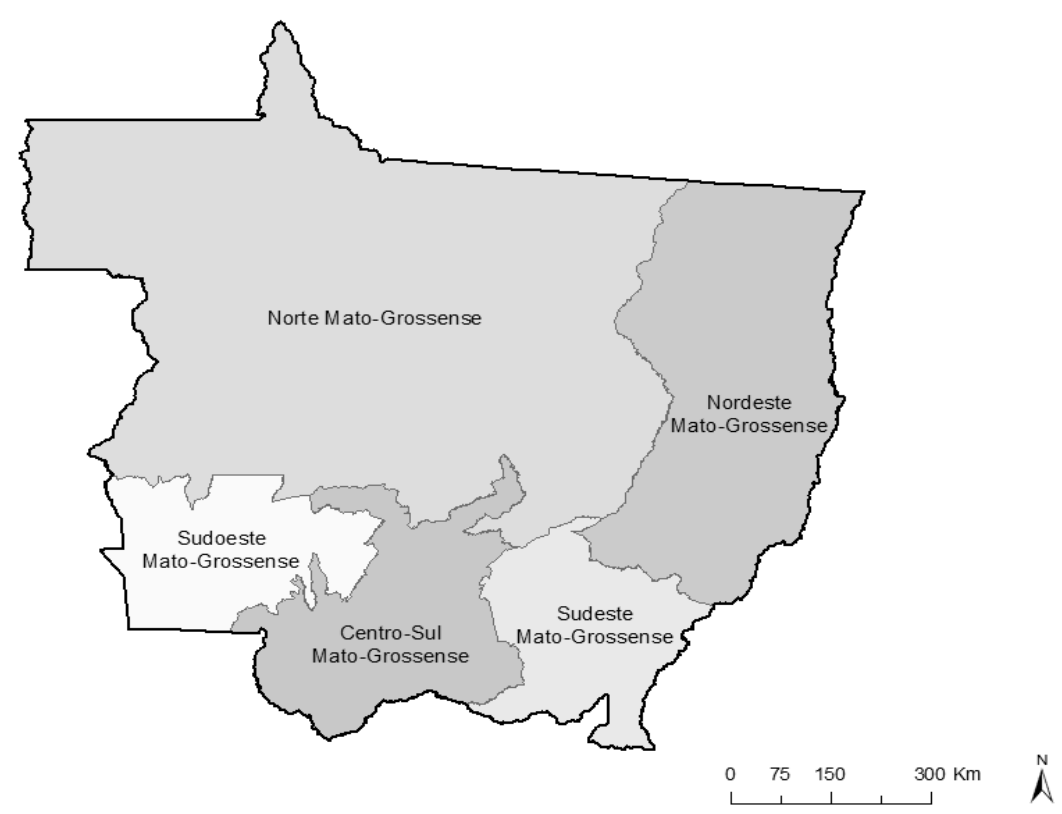

Figura 1. Mapa da localização das mesorregiões do estado Mato-Grossense Fonte: IBGE (2015)

$\mathrm{Na}$ Tabela 1 foi possível observar a quantidade de municípios em cada mesorregião bem como as produtividades médias e os coeficientes de variação. Onde o CV para a produtividade de milho variou de $11,4 \%$ à $25,3 \%$, e para a produtividade de soja variou de $1,85 \%$ à $8,38 \%$. 
Tabela 1. Produtividade e coeficiente de variação [CV] de milho e soja para cada mesorregião do estado de Mato Grosso

\begin{tabular}{cccccc}
\hline \multirow{2}{*}{ Mesorregiões } & \multirow{2}{*}{$\begin{array}{c}\mathrm{N}^{0} \text { de } \\
\text { municípios }\end{array}$} & \multicolumn{2}{c}{ Milho } & \multicolumn{2}{c}{ Soja } \\
\cline { 3 - 6 } & & Produtividade & $\mathrm{CV}$ & Produtividade & $\mathrm{CV}$ \\
\hline \multirow{2}{*}{ Centro-sul } & 6 & 3607 & 12,6 & 2921 & $---\mathrm{kg} \mathrm{ha}^{-1}-----$ \\
Nordeste & 14 & 3477 & 17,0 & 2917 & 5,25 \\
Norte & 49 & 3504 & 14,0 & 2942 & 5,53 \\
Sudeste & 17 & 4370 & 11,4 & 2949 & 3,37 \\
Sudoeste & 6 & 2959 & 25,3 & 2792 & 8,38 \\
\hline
\end{tabular}

Fonte: Resultados originais da pesquisa

Inicialmente, pode-se ter uma ideia do risco associado considerando as mesorregiões, entretanto sabe-se que os municípios foram reunidos geograficamente, desconsiderando à similaridade produtiva entre estes. Nesse caso, as operadoras irão trabalhar em uma situação arriscada. Por isso a necessidade de agrupamento dos municípios quanto à sua similaridade produtiva e a busca de taxas de risco (CV) menores.

Assim, o método de agrupamento utilizado reuniu os municípios em sete clusters. O número de clusters foi determinado pelo corte realizado no dendograma (Figura 2).

$\mathrm{Na}$ utilização dos dois métodos conjuntamente para a formação de grupos de municípios, o método Ward (dendograma) definiu com mais eficiência a quantidade de agrupamentos que devem ser utilizados, enquanto que o método K-means classificou de forma mais adequada os municípios dentro dos agrupamentos. As cidades que compõe cada cluster estão descritas na Tabela 2. 


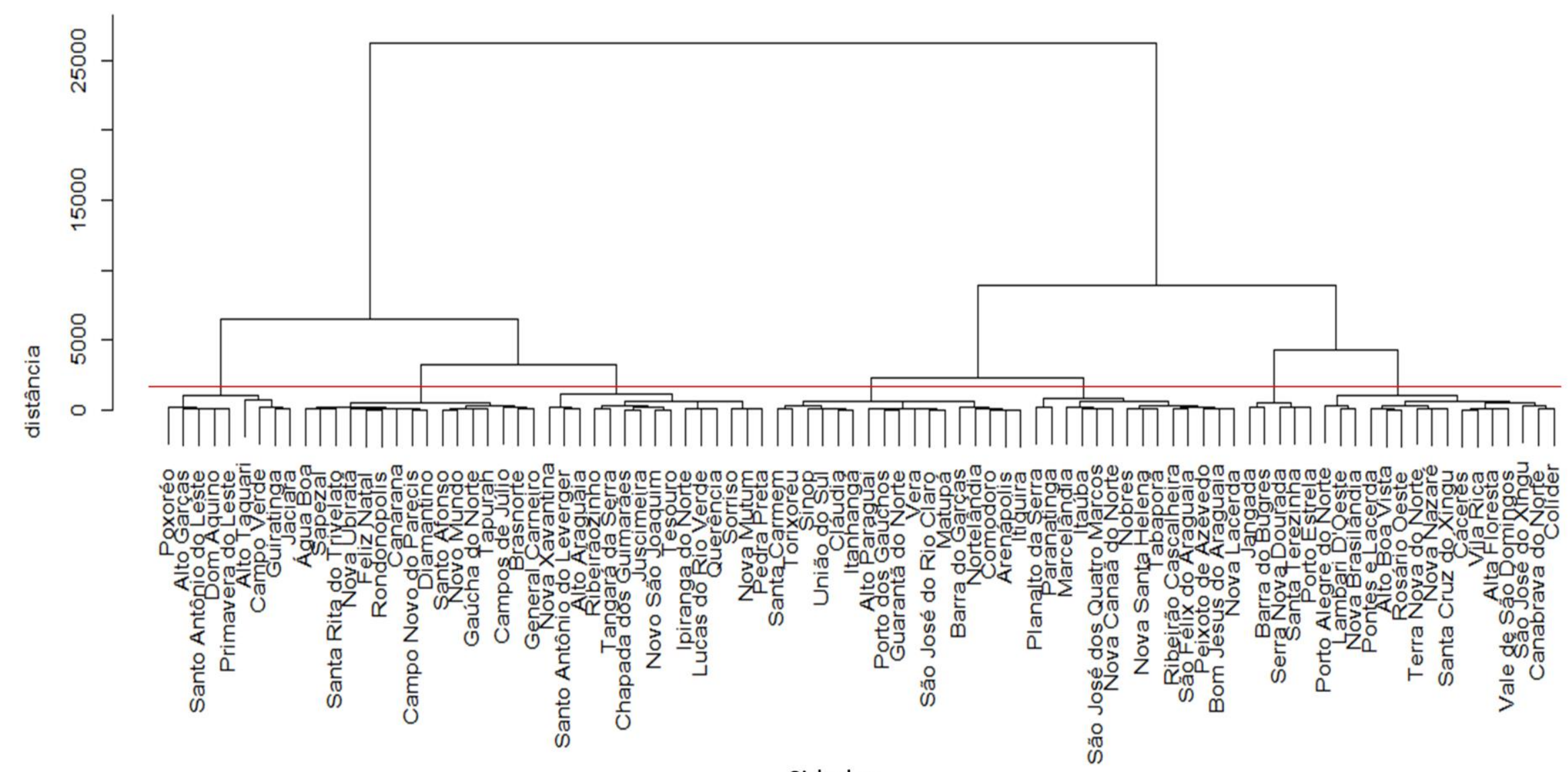

\section{Cidades}

Figura 2. Dendograma obtido para os 92 municípios do Estado de Mato Grosso considerando a produtividade de soja e milho, em kg ha. O traço em vermelho indica o corte realizado para a quantificação do número de clusters

Fonte: Resultados originais da pesquisa 
Tabela 2. Alocação das 92 cidades nos sete clusters, e a mesorregião correspondente à cada cidade

(continua)

\begin{tabular}{|c|c|c|}
\hline Número do cluster & Nome da cidade & Mesorregião \\
\hline 1 & Cáceres & Centro-Sul \\
\hline 1 & São José do Xingu & Nordeste \\
\hline 1 & Canabrava do Norte & Nordeste \\
\hline 1 & Bom Jesus do Araguaia & Nordeste \\
\hline 1 & Vila Rica & Nordeste \\
\hline 1 & Alto Boa Vista & Nordeste \\
\hline 1 & Ribeirão Cascalheira & Nordeste \\
\hline 1 & Nova Nazaré & Nordeste \\
\hline 1 & Marcelândia & Norte \\
\hline 1 & São José dos Quatro Marcos & Norte \\
\hline 1 & Itaúba & Norte \\
\hline 1 & Colíder & Norte \\
\hline 1 & Nova Canaã do Norte & Norte \\
\hline 1 & Nova Santa Helena & Norte \\
\hline 1 & Peixoto de Azevedo & Norte \\
\hline 1 & Tabaporã & Norte \\
\hline 1 & Rosário Oeste & Norte \\
\hline 1 & Nobres & Norte \\
\hline 1 & Alta Floresta & Norte \\
\hline 1 & Santa Cruz do Xingu & Norte \\
\hline 1 & São Félix do Araguaia & Norte \\
\hline 1 & Terra Nova do Norte & Norte \\
\hline 1 & Planalto da Serra & Norte \\
\hline 1 & Lambari D'Oeste & Norte \\
\hline 1 & Paranatinga & Norte \\
\hline 1 & Nova Brasilândia & Norte \\
\hline 1 & Nova Lacerda & Sudoeste \\
\hline 1 & Pontes e Lacerda & Sudoeste \\
\hline 1 & Vale de São Domingos & Sudoeste \\
\hline 2 & Chapada dos Guimarães & Centro-Sul \\
\hline 2 & Nova Xavantina & Nordeste \\
\hline 2 & Santo Antônio do Leverger & Norte \\
\hline 2 & Juscimeira & Sudeste \\
\hline 2 & Alto Araguaia & Sudeste \\
\hline 3 & Porto Alegre do Norte & Nordeste \\
\hline 3 & Serra Nova Dourada & Norte \\
\hline 3 & Santa Terezinha & Norte \\
\hline 3 & Jangada & Norte \\
\hline 3 & Barra do Bugres & Sudoeste \\
\hline 3 & Porto Estrela & Sudoeste \\
\hline 4 & Novo São Joaquim & Nordeste \\
\hline 4 & Água Boa & Nordeste \\
\hline 4 & Ipiranga do Norte & Norte \\
\hline 4 & Lucas do Rio Verde & Norte \\
\hline 4 & Sorriso & Norte \\
\hline 4 & Querência & Norte \\
\hline 4 & Nova Mutum & Norte \\
\hline 4 & Nova Ubiratã & Norte \\
\hline 4 & Sapezal & Norte \\
\hline
\end{tabular}


Tabela 2. Alocação das 92 cidades nos sete clusters, e a mesorregião correspondente à cada cidade

(conclusão)

\begin{tabular}{|c|c|c|}
\hline Número do cluster & Nome da cidade & Mesorregião \\
\hline 4 & Santa Rita do Trivelato & Norte \\
\hline 4 & Pedra Preta & Sudeste \\
\hline 4 & Tesouro & Sudeste \\
\hline 4 & Ribeirãozinho & Sudeste \\
\hline 4 & Tangará da Serra & Sudoeste \\
\hline 5 & Santo Afonso & Centro-Sul \\
\hline 5 & Alto Paraguai & Centro-Sul \\
\hline 5 & Nortelândia & Centro-Sul \\
\hline 5 & Arenápolis & Centro-Sul \\
\hline 5 & Canarana & Nordeste \\
\hline 5 & Barra do Garças & Nordeste \\
\hline 5 & Santa Carmem & Norte \\
\hline 5 & Gaúcha do Norte & Norte \\
\hline 5 & União do Sul & Norte \\
\hline 5 & Sinop & Norte \\
\hline 5 & Feliz Natal & Norte \\
\hline 5 & Tapurah & Norte \\
\hline 5 & Porto dos Gaúchos & Norte \\
\hline 5 & Guarantã do Norte & Norte \\
\hline 5 & Vera & Norte \\
\hline 5 & Novo Mundo & Norte \\
\hline 5 & Cláudia & Norte \\
\hline 5 & Campo Novo do Parecis & Norte \\
\hline 5 & São José do Rio Claro & Norte \\
\hline 5 & Itanhangá & Norte \\
\hline 5 & Diamantino & Norte \\
\hline 5 & Matupá & Norte \\
\hline 5 & Brasnorte & Norte \\
\hline 5 & Comodoro & Norte \\
\hline 5 & Campos de Júlio & Norte \\
\hline 5 & Torixoréu & Sudeste \\
\hline 5 & Rondonópolis & Sudeste \\
\hline 5 & General Carneiro & Sudeste \\
\hline 5 & Itiquira & Sudeste \\
\hline 6 & Santo Antônio do Leste & Nordeste \\
\hline 6 & Poxoréo & Sudeste \\
\hline 6 & Guiratinga & Sudeste \\
\hline 6 & Dom Aquino & Sudeste \\
\hline 6 & Primavera do Leste & Sudeste \\
\hline 6 & Jaciara & Sudeste \\
\hline 6 & Alto Garças & Sudeste \\
\hline 7 & Campo Verde & Sudeste \\
\hline 7 & Alto Taquari & Sudeste \\
\hline
\end{tabular}

Fonte: Resultados originais da pesquisa 
A Figura 2 ilustra os municípios alocados em cada cluster, ressalvo uma informação de que municípios muito pequenos não aparecem destacados no mapa, mas foram considerados no estudo.

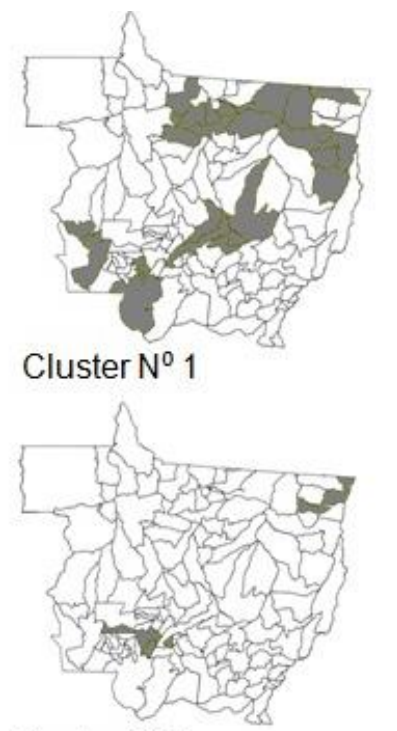

Cluster $\mathrm{N}^{0} 3$

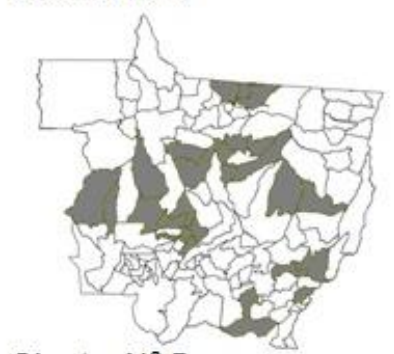

Cluster $\mathrm{N}^{0} 5$

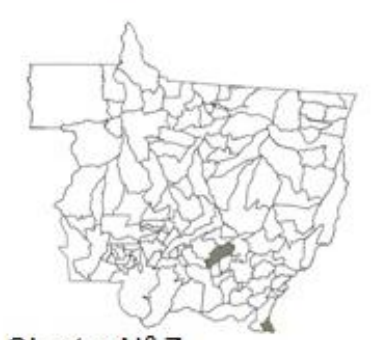

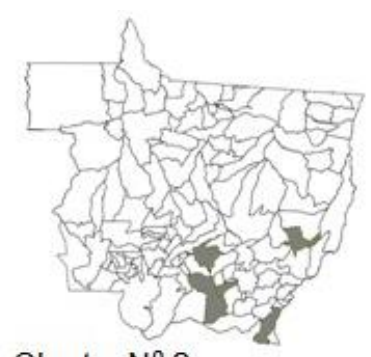

Cluster $\mathrm{N}^{0} 2$

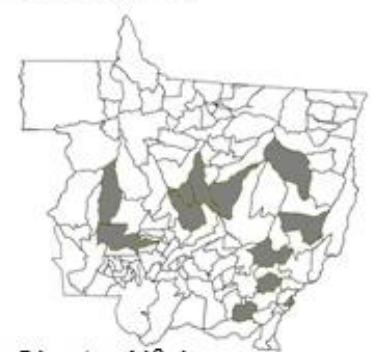

Cluster $\mathrm{N}^{\circ} 4$

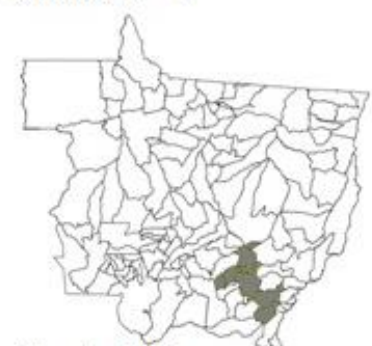

Cluster $\mathrm{N}^{0} 6$

Cluster $\mathrm{N}^{0} 7$

Figura 2. Alocação dos municípios do Estado de Mato Grosso em cada um dos sete clusters

Fonte: Resultados originais da pesquisa

A Tabela 3 apresenta as características de cada cluster considerando dados de produtividade de grãos de milho. O CV para a produtividade de milho variou de $1,63 \%$ e 10,2\%, valores bem menores comparados ao da Tabela 1, quando os municípios foram agrupados geograficamente desconsiderando o potencial produtivo dos municípios. 
Tabela 3. Produtividade média (em $\mathrm{kg} \mathrm{ha}^{-1}$ ) de milho, número de municípios, desvio padrão da média [s] e coeficiente de variação [CV] de cada cluster formado

\begin{tabular}{|c|c|c|c|c|}
\hline $\mathrm{N}^{\circ}$ & Produtividade média & $\mathrm{N}^{\circ}$ & $s$ & $\mathrm{CV}$ \\
\hline do cluster & - & de municípios & ---- kg ha-1 ---- & ---- \% --- \\
\hline 1 & 3132 & 29 & 180,59 & 5,76 \\
\hline 2 & 4239 & 5 & 69,09 & 1,63 \\
\hline 3 & 2360 & 6 & 241,65 & 10,2 \\
\hline 4 & 4146 & 14 & 147,92 & 3,57 \\
\hline 5 & 3686 & 29 & 158,68 & 4,31 \\
\hline 6 & 4657 & 7 & 120,64 & 2,59 \\
\hline 7 & 5170 & 2 & 276,93 & 5,36 \\
\hline
\end{tabular}

Fonte: Resultados originais da pesquisa

As seguradoras podem trabalhar tranquilamente nos municípios do cluster número 2 (Chapada dos Guimarães, Nova Xavantina, Santo Antônio do Leverger, Juscimeira e Alto Araguaia) e 6 (Santo Antônio do Leste, Poxoréo, Guiratinga, Dom Aquino, Primavera do Leste, Jaciara e Alto Garças) onde as produtividades médias (4239 $\mathrm{kg} \mathrm{ha}^{-1}$ e $4657 \mathrm{~kg} \mathrm{ha}^{-1}$, respectivamente) são maiores inclusive os riscos associados são considerados baixos.

A menor produtividade média foi do cluster número $3\left(2360 \mathrm{~kg} \mathrm{ha}^{-1}\right)$, onde constam seis municípios e cuja variabilidade média foi considerada alta comparativamente aos outros clusters. Ou seja, a magnitude do risco foi considerada elevada. Essa é uma informação importante para a tomada de decisão das seguradoras, pois sugere que as taxas de prêmio desses municípios serão maiores comparadas aos municípios de baixo risco. Além das taxas de prêmio, os níveis de cobertura também devem variar, por exemplo, coberturas menores para os municípios de alto risco e vice-versa.

Tabela 4. Produtividade média (em $\mathrm{kg} \mathrm{ha}^{-1}$ ) de soja, número de municípios do estado de Mato Grosso, desvio padrão da média ( $s$ ) e coeficiente de variação [CV] de cada cluster formado

\begin{tabular}{|c|c|c|c|c|}
\hline $\mathrm{N}^{0}$ & Produtividade média & $\mathrm{N}^{0}$ & $s$ & $\mathrm{CV}$ \\
\hline do cluster & 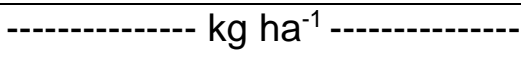 & de municípios & -- kg ha-1 - & ------ \% ------ \\
\hline 1 & 2937 & 29 & 156,4 & 5,33 \\
\hline 2 & 2742 & 5 & 117,8 & 4,30 \\
\hline 3 & 2583 & 6 & 105,8 & 4,10 \\
\hline 4 & 3035 & 14 & 83,5 & 2,75 \\
\hline 5 & 2955 & 29 & 68,8 & 2,33 \\
\hline 6 & 2983 & 7 & 62,6 & 2,10 \\
\hline 7 & 2981 & 2 & 84,8 & 2,85 \\
\hline
\end{tabular}

Fonte: Resultados originais da pesquisa 
Para a soja, as informações estão contidas na Tabela 4. Foi observado que o CV variou de $2,10 \%$ e 5,33\%, essa amplitude foi menor comparada àquela encontrada na Tabela 1.

Observou-se uma situação mais homogênea do que no milho, já que a variação entre os grupos foi baixa bem como a magnitude do risco associado à cada cluster. Essa baixa variação média pode estar associada a não ocorrência de ferrugem asiática da soja (a doença mais preocupante da soja no Brasil) no estado do Mato Grosso até o momento, o que tende a assegurar a produtividade média da soja em um patamar elevado e livre de oscilações ao longo dos anos. O cluster com o maior valor de CV foi o número 1 , entretanto o grupo não apresentou a menor produtividade. $O$ grupo 3 foi aquele que novamente apresentou média de produtividade baixa e englobou os municípios citados na Tabela 5.

Tabela 5. Mesorregiões, municípios, produtividade de milho e soja e coeficiente de variação [CV] de cada município

\begin{tabular}{cccccc}
\hline \multirow{2}{*}{ Mesorregiões } & Municípios do & \multicolumn{2}{c}{ Milho } & \multicolumn{2}{c}{ Soja } \\
\cline { 3 - 6 } & cluster $\mathrm{n}^{0} 3$ & Produtividade & CV & Produtividade & CV \\
\hline \multirow{2}{*}{ Nordeste } & Porto Alegre do Norte & 2733 & 45,8 & 2728 & 16,1 \\
Norte & Serra Nova Dourada & 2345 & 18,7 & 2699 & 20,6 \\
Norte & Santa Terezinha & 2492 & 54,1 & 2570 & 22,4 \\
Norte & Jangada & 2186 & 21,6 & 2510 & 26,7 \\
Sudoeste & Barra do Bugres & 2036 & 15,5 & 2516 & 12,31 \\
Sudoeste & Porto Estrela & 2366 & 29,3 & 2475 & 14,27 \\
\hline
\end{tabular}

Fonte: Resultados originais da pesquisa

A tomada de decisão por parte das seguradoras não deveria considerar o valor de CV isoladamente, já que esse valor pode estar associado ao aumento da produtividade ao longo dos anos provocando variabilidade das médias de produtividade. Entretanto, Ozaki (2009) considera que os municípios que apresentarem valores de risco $(\mathrm{CV})$ maiores em relação ao risco médio total (CV milho: $23 \%$ e CV soja: 9,38\%), devem ser excluídos das carteiras das seguradoras. Nesse estudo, 61 municípios apresentam o risco individual maior do que o risco agregado, considerando apenas o CV milho, é um número de municípios muito alto e a exclusão não deve ocorrer sem que se considere o risco agregado na produção de soja, bem como as características dos produtores da região.

Outra alternativa para as seguradoras é a possibilidade de direcionar os recursos dos programas estaduais de subvenção ao seguro rural para esses 
municípios. No último relatório do MAPA (2015), no Mato Grosso para o milho $1^{\underline{a}}$ safra, o percentual de subvenção do seguro rural foi de $40 \%$ sobre o valor do prêmio estipulado em apólice, para o milho $2^{2}$ safra foi $60 \%$ e para a soja foi $56 \%$.

\section{Conclusões}

A quantificação municipalizada do risco é fundamental para que as seguradoras possam trabalhar com risco diversificado e precificar adequadamente os contratos.

O risco agregado é mais preocupante quando se considera a produtividade do milho nas propriedades em comparação à produtividade de soja.

As seguradoras podem avançar em áreas agrícolas importantes, mas que até então não atraíram as seguradoras devido ao histórico elevado de perdas. Entretanto, existe a opção de contratar prêmios de valores mais baixos e aderir ao programa de subvenção ao seguro rural.

\section{Referências}

Berlato, M.A.; Fontana, D.C. 1999. Variabilidade interanual de precipitação pluvial e rendimento de soja no Estado do Rio Grande do Sul. Revista Brasileira de Agrometeorologia 7: 119-125.

Compania Nacional de Abastecimento [CONAB]. 2016. Levantamento SAFRA. Disponível em: <http://www.conab.gov.br/conoteudos.php?a=1253\&>. Acesso em: 22 abr. 2016

Everitt, B.S.; Landau, S.; Leese, M.; Stahl, D. 2011. Cluster Analysis. Nova Jersey: Wiley, New Jersey, USA.

Favarin, J.L.; Oliveira, A.C.B. de. 2013. Zoneamento agrícola: há o que se considerar. Visão Agrícola 8(12): 88-89.

Frei, F. 2006. Introdução à análise de agrupamentos: Teoria e prática. Editora UNESP, São Paulo, São Paulo, Brasil.

Hair Jr., J.F.; Black, W.C.; Babin, B.J.; Anderson, R.E.; Tatahn, R.L. 2005. Análise multivariada de dados. 5ed. Bookman, Porto Alegre, Rio Grande do Sul, Brasil.

Instituto Brasileiro de Geografia e Estatística [IBGE]. 2015. Pesquisa Pecuária Municipal. Disponível em: <www.sidra.ibge.gov.br>. Acesso em: 02 nov. 2015.

Instituto Brasileiro de Geografia e Estatística [IBGE]. 2016. Estatística da Produção Agrícola: dezembro de 2016. Disponível em: <www.sidra.ibge.gov.br>. Acesso em: 01 fev. 2017.

Manly, B.F.J. 1986. Multivariate statistical methods: a primer. Chapman and Hall, London, Great Britain. 
Ministério da Agricultura Pecuária e Abastecimento. [MAPA] 2015. Resultado Geral PSR. Disponível em:

<http://www.agricultura.gov.br/arq_editor/file/Seguro\%20Rural/PSR\%20-

\%20Resultado\%20Geral\%202015.pdf>. Acesso em: 02 maio 2016.

Ministério da Agricultura Pecuária e Abastecimento [MAPA] 2016. Seguro Rural. Disponível em: <www.agricultura.gov.br>. Acesso em: 02 maio 2016.

Musshoff, O.; Odening, M.; Xu, W. 2011. Management of climate risks in agriculture will weather derivatives permeate? Applied economics 43(9): 1067-1077.

Ozaki, V.A. 2007. O papel do seguro na gestão do risco agrícola e os empecilhos para o seu desenvolvimento. Revista Brasileira de Risco e Seguro 2(4): 75-92.

Ozaki, V.A. 2008. Em busca de um novo paradigma para o seguro rural no Brasil. Revista de Economia e Sociologia Rural 46(1): 97-119.

Ozaki, V.A. 2009. Análise e quantificação do risco para a gestão eficiente do portfólio agrícola das seguradoras. Revista de Economia e Sociologia Rural 47(3): 549-567.

Pohlmann, M.C. 2007. Análise de conglomerados. In: Corrar, L.J.; Paulo, E.; Dias Filho, J.M. Análise multivariada para os cursos de administração, ciências contábeis e economia. Atlas, São Paulo, São Paulo, Brasil.

Ullah, R.; Damien, J.; Ganesh, P.S.; Shobhakar, D. 2015. Managing catastrophic risks in agriculture: Simultaneous adoption of diversification and precautionary savings. International Journal of Disaster Risk Reduction 12: 268-277. 